\title{
Emprego de um Catalisador de Cromo (III) Contendo 0 Ligante N,N'-di-t-Bu-2,11-diaza [3.3] (2,6)-piridinofano na Polimerização de Etileno e de Propileno
}

\author{
Simoni M. P. Meneghetti \\ Politeno Indústria e Comércio S.A., Bahia \\ Pierre Lutz \\ Institut Charles Sadron, Strasbourg, França \\ Jacky Kress \\ Université Louis Pasteur, Strasbourg, França
}

Resumo: Este trabalho trata da síntese e caracterização de um complexo de $\mathrm{Cr}(\mathrm{III})$ contendo o ligante N,N'-di-t-Bu-2,11diaza [3.3] (2,6)-piridinofano $\left(t-\mathrm{Bu}-\mathrm{N}_{4} \mathrm{CrCl}_{2}{ }^{+} \mathrm{Cl}\right)$ e posterior avaliação catalítica na polimerização de etileno e propileno, sob várias condições de reação, em presença de $\mathrm{MAO}$ (metilaluminoxana) ou $\mathrm{Et}_{3} \mathrm{Al}_{2} \mathrm{Cl}_{3}$ (sesquicloreto de etilalumínio EASC), como cocatalisadores. O emprego do sistema catalítico $t$ - $\mathrm{Bu}-\mathrm{N}_{4} \mathrm{CrCl}_{2}^{+} \mathrm{Cl}^{-} / \mathrm{Et}_{3} \mathrm{Al}_{2} \mathrm{Cl}_{3}$ produziu copolímeros de etileno-propileno e de polietilenos, com bons rendimentos. A caracterização desses polímeros, através de várias técnicas analíticas, mostrou que os mesmos são essencialmente lineares e de alta massa molar.

Palavras-chave: Catálise, poliolefinas, cromo(III), ligantes macrocíclicos, diazapiridinofano.

\section{Use of the chromium (III) catalyst bearing N,N'-di-t-Bu-2, 11-diaza[3.3] (2,6)-pyridinophane ligand in polymerisation of} ethylene and propylene

Abstract: Chromium (III) complex bearing N,N'- di-t-Bu-2, 11-diaza[3.3] (2,6)-pyridinophane ligand $\left(t-\mathrm{Bu}_{-} \mathrm{N}_{4} \mathrm{CrCl}_{2}^{+} \mathrm{Cl}^{-}\right)$ was synthesized and characterized. This complex was tested in ethylene and propylene polymerisation, under several reaction conditions, in the presence of $\mathrm{MAO}$ (metilaluminoxane) or $\mathrm{Et}_{3} \mathrm{Al}_{2} \mathrm{Cl}_{3}$ (ethyl aluminium sesquichloride), as cocatalysts. The use of the catalytic system $t-\mathrm{Bu}_{-} \mathrm{N}_{4} \mathrm{CrCl}_{2}^{+} \mathrm{Cl} / \mathrm{Et}_{3} \mathrm{Al}_{2} \mathrm{Cl}_{3}$ led to ethylene-propylene copolymers and polyethylene, with good yields. The polymers thus produced display high molar masses and narrow polydispersities.

Keywords: Catalysis, polyolefins, chromium (III), macrocyclic ligand, pyridinophane.

\section{Introdução}

A catálise de polimerização de olefinas, em presença de cromo, é um campo de pesquisa ainda em desenvolvimento $^{[1-5]}$. Os mecanismos envolvidos na catálise heterogênea (Processos Phillips e Union Carbide) não estão completamente estabelecidos e estudos em meio homogêneo, principalmente em presença de complexos moleculares de estrutura definida, podem fornecer informações valiosas do ponto de vista mecanístico ${ }^{[6-10]}$.

Ligantes nitrogenados têm se revelado bastante versáteis na estabilização de complexos com potencial catalítico na polimerização de olefinas ${ }^{[11]}$. Porém, a possibilidade de se utilizar ligantes nitrogenados macrocíclicos, como os diazapiridinofanos, coordenados a metais de transição, para a polimerização de olefinas, ainda não havia sido investigada. Nossos trabalhos mostraram a potencialidade desses ligantes em estudos mecanísticos e de fluxionalidade de vários complexos ${ }^{[12,13]}$.

\section{Experimental}

\section{Materiais}

A síntese e caracterização do complexo de $\mathrm{Cr}(\mathrm{III})$ contendo o ligante N,N'-di-t-Bu-2,11-diaza [3.3] $(2,6)$ piridinofano ${ }^{[13]}$, foi realizada sob atmosfera inerte utilizando técnicas de Schlenk (manipulação sob atmosfera inerte).

O ligante N,N'-di-t-Bu-2,11-diaza [3.3] (2,6)-piridinofano foi sintetizado pela reação entre o 2,6-bis(bromometil)piridina e a $t$-butilamina, em duas etapas, conforme esquema 1. O mesmo foi purificado por recristalização (resfriamento de uma solução em etanol), sob a forma de cristais brancos,com rendimento de $54 \%{ }^{[12,13]}$.

Autor para correspondência: Simoni M. P. Meneghetti, Politeno Indústria e Comércio S.A., Rua Benzeno, no 2391, Pólo Petroquímico, CEP: 42810-000, Camaçari, BA. E-mail: simoni.meneghethi@politeno.com.br 

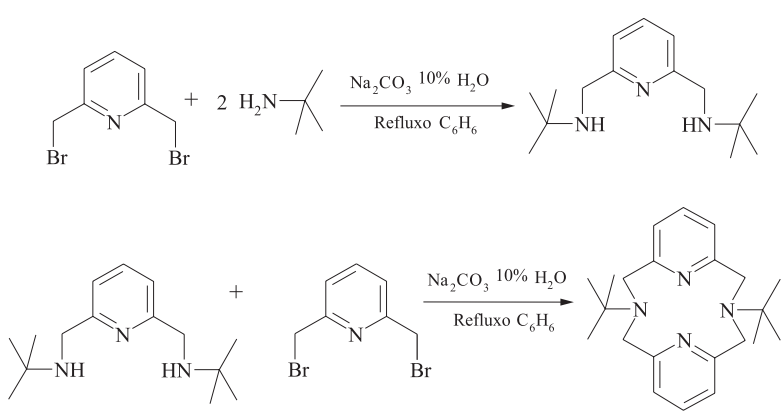

O complexo de $\mathrm{Cr}$ (III) contendo o ligante N,N'-di-t-Bu-2,11diaza [3.3] (2,6)-piridinofano $\left(t-\mathrm{Bu}-\mathrm{N}_{4} \mathrm{CrCl}_{2}{ }^{+} \mathrm{Cl}^{-}\right)$foi sintetizado pela reação entre o ligante N,N'-di-t-Bu-2,11-diaza [3.3] (2,6)-piridinofano $\left(t-\mathrm{Bu}-\mathrm{N}_{4}\right)$ e o precursor $(\mathrm{THF})_{3} \mathrm{CrCl}_{3}$, em THF (tetraidrofurano) como solvente, conforme reação 1, abaixo.

$$
(\mathrm{THF})_{3} \mathrm{CrCl}_{3}+t \text {-Bu-N } \mathrm{N}_{4} \stackrel{\mathrm{THF}}{\longrightarrow}\left(t-\mathrm{Bu}_{-} \mathrm{N}_{4}\right) \mathrm{CrCl}_{2}^{+} \mathrm{Cl}^{-}+3 \mathrm{THF}
$$

Todos os solventes utilizados foram secos segundo procedimentos descritos na literatura ${ }^{[14]}$. O etileno e o propileno utilizados nas reações de polimerização foram adquiridos com pureza 99,9\% (Air Liquids).

\section{Reação de polimerização}

Numa reação de polimerização típica, o experimento é realizado à temperatura de trabalho selecionada, num reator Buchi de $250 \mathrm{~mL}$, equipado com agitação mecânica, submetido a ciclos de purga com argônio e aplicação de vácuo. Todas as manipulações são realizadas empregando técnicas de Schlenk. Após adição de tolueno e catalisador o reator é pressurizado com etileno e/ou propileno e o cocatalisador é adicionado. Após o tempo de reação estabelecido, o reator é despressurizado e o polímero formado é precipitado em $\mathrm{MeOH} / \mathrm{HCl}$ e seco à vácuo até peso constante.

\section{Caracterização dos Polímeros}

Os polímeros foram caracterizados por Espectroscopia de Ressonância Magnética Nuclear (equipamentos Bruker AC-200, AC-300 e AM-400), Espectroscopia na região do Infravermelho Médio (FTIR Bomen MB 155) e SEC (cromatografia de exclusão de tamanho) a $140{ }^{\circ} \mathrm{C}$ (Waters 150C, TCB (triclorobenzeno), detector índice de refração (padrões de PE linear) e DSC (calorimetria diferencial de varredura) (DSC4 Perkin Elmer).

\section{Resultados e Discussão}

A solução formada pelo complexo (THF) ${ }_{3} \mathrm{CrCl}_{3} \mathrm{em}$ THF apresenta uma coloração rosa intensa e, após a reação, a formação de um sólido de coloração rosa pálida indicou a presença do complexo (1). Este sólido formado, instável ao ar, apresentou-se extremamente insolúvel em vários solventes testados $\left(\mathrm{CH}_{2} \mathrm{Cl}_{2}, \mathrm{CHCl}_{3}, \mathrm{MeCN}\right.$, tolueno, etc. $)$ e esta insolubilidade impediu uma caracterização mais detalhada por $\mathrm{RMN}{ }^{1} \mathrm{H}$ ou a obtenção de cristais para caracterização por difração de Raio X.
Porém, a caracterização por espectroscopia no infravermelho indicou a substituição das moléculas de THF no complexo precursor pelas moléculas do ligante, principalmente pelo desaparecimento da banda de absorção a $845 \mathrm{~cm}^{-1}$, característica das moléculas de THF. As bandas das ligações C-Cl (400-300 $\left.\mathrm{cm}^{-1}\right)$ do complexo inicial sofreram modificações: 3 bandas existentes no complexo precursor $\left((\mathrm{THF})_{3} \mathrm{CrCl}_{3}\right)$ deram lugar a apenas 2 bandas, em concordância com a estrutura $t$ - $\mathrm{Bu}-\mathrm{N}_{4} \mathrm{CrCl}_{2}^{+} \mathrm{Cl}^{-}$.

$\mathrm{O}$ caráter iônico do complexo, na estrutura proposta, justifica também a insolubilidade observada para o mesmo. Os resultados de análise elementar confirmam, com boa aproximação, a formação do complexo [\% determinado (calculado) para $\mathrm{C}_{22} \mathrm{H}_{32} \mathrm{~N}_{4} \mathrm{CrCl}_{3}: \mathrm{C}: 48,83(51,71), \mathrm{H}: 7,08(6,27), \mathrm{N}$ : $8,50(10,97)]$.

\section{Homopolimerização do Etileno e Caracterização dos Polietilenos Obtidos}

Os testes catalíticos indicam que o complexo $t$-Bu$\mathrm{N}_{4} \mathrm{CrCl}_{2}{ }^{+} \mathrm{Cl}^{-}$é ativo em presença de etileno conduzindo à formação de polietilenos. Os principais resultados dos testes de homopolimerização estão apresentados na Tabela 1.

Como cocatalisadores foram testados o $\mathrm{Et}_{3} \mathrm{Al}_{2} \mathrm{Cl}_{3}$ (sesquicloreto de etil alumínio) e o MAO (metilaluminoxana). A atividade do sistema $t-\mathrm{Bu}-\mathrm{N}_{4} \mathrm{CrCl}_{2}^{+} \mathrm{Cl}^{-} / \mathrm{Et}_{3} \mathrm{Al}_{2} \mathrm{Cl}_{3}$ é mais alta que a do sistema $t$ - $\mathrm{Bu}-\mathrm{N}_{4} \mathrm{CrCl}_{2}^{+} \mathrm{Cl}^{-} / \mathrm{MAO}$. O fator que determina esse comportamento é difícil de estabelecer, pois vários aspectos devem ser analisados num processo de ativação. Além do poder de abstração de halogêneos ou o poder de alquilação de um cocatalisador, a estrutura das espécies catalíticas formadas deve ser considerada. Neste contexto cabe destacar que o tipo de contra-íon formado e a energia de associação do par iônico podem ter papéis fundamentais ${ }^{[15]}$. Uma possibilidade é atribuir esta maior eficácia do EASC como cocatalisador ao seu poder alquilante e sua acidez de Lewis superiores, quando comparado ao MAO.

A variação da razão $\mathrm{Et}_{3} \mathrm{Al}_{2} \mathrm{Cl}_{3} /$ catalisador foi avaliada e a

Tabela 1. Polimerização de etileno em presença de $t-\mathrm{Bu}-\mathrm{N}_{4} \mathrm{CrCl}_{2}{ }^{+} \mathrm{Cl}$

\begin{tabular}{ccccccc}
\hline & $\begin{array}{c}\mathbf{T} \\
\left({ }^{\circ} \mathbf{C}\right)\end{array}$ & $\begin{array}{c}\mathbf{A l} / \mathbf{C r} \\
(\mathbf{1})\end{array}$ & $\begin{array}{c}\text { Rdt. } \\
(\mathbf{g})\end{array}$ & $\begin{array}{c}\text { Atividade } \\
(\mathbf{2})\end{array}$ & $\begin{array}{c}\mathbf{T}_{\mathbf{f}} \\
(\mathbf{3})\end{array}$ & $\begin{array}{c}\mathbf{X}_{\mathbf{c}} \\
\mathbf{( 4 )}\end{array}$ \\
\hline 1 & 20 & $180 \mathrm{MAO}$ & 1,1 & 3150 & 139,0 & 76 \\
2 & 20 & $80 \mathrm{Et}_{3} \mathrm{Al}_{2} \mathrm{Cl}_{3}$ & 11,9 & 34000 & 139,3 & 66 \\
3 & 20 & $40 \mathrm{Et}_{3} \mathrm{Al}_{2} \mathrm{Cl}_{3}$ & 12,0 & 34300 & 140,0 & 61 \\
4 & 20 & $20 \mathrm{Et}_{3} \mathrm{Al}_{2} \mathrm{Cl}_{3}$ & 10,0 & 28600 & 139,6 & 61 \\
5 & 40 & $80 \mathrm{Et}_{3} \mathrm{Al}_{2} \mathrm{Cl}_{3}$ & 2,1 & 5950 & 140,1 & 66 \\
6 & 60 & $40 \mathrm{Et}_{3} \mathrm{Al}_{2} \mathrm{Cl}_{3}$ & 1,6 & 6850 & 140,5 & 73 \\
\hline
\end{tabular}

Condições de polimerização: $100 \mathrm{~mL}$ tolueno, $2 \mathrm{~h}, 0,025 \mathrm{mmol}$ de catalisador, 7 bar de pressão de etileno.

(1) Razão molar $\mathrm{Al} / \mathrm{Cr}$;

(2) g polímero/(h.bar.mol catalisador);

(3) Temperatura de fusão determinada por DSC;

(4) \% de cristalinidade determinada por DSC considerando um calor de fusão de um polietileno $100 \%$ cristalino em $64,5 \mathrm{cal} / \mathrm{g}$. 


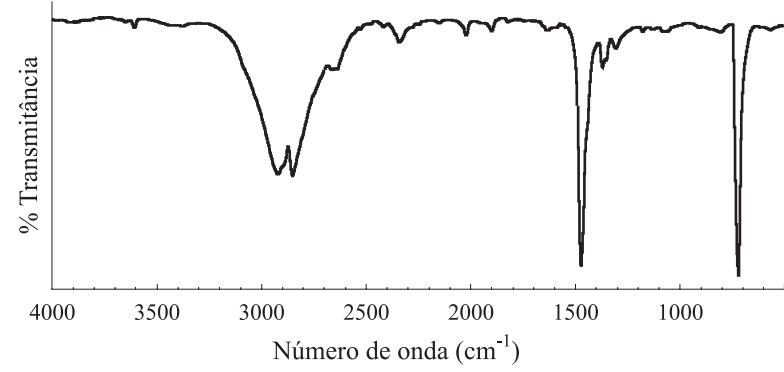

Figura 1. Espectro de absorção na região do infravermelho médio para o polietileno obtido no teste catalítico 6

atividade foi otimizada a uma razão de aproximadamente 40/1 (testes 2,3 e 4 ).

O aumento da temperatura de polimerização (testes 3, 5 e 6) resultou na diminuição do rendimento da reação, devido, provavelmente, a uma rápida desativação do sistema catalítico.

Os polietilenos (PE) obtidos foram caracterizados por espectroscopia no infravermelho, DSC e SEC alta temperatura (em alguns casos). Os resultados de DSC podem ser vistos na Tabela 1. Estas caracterizações revelaram que estes PE são de alta massa molecular [Teste $3\left(\mathrm{M}_{\mathrm{w}}=896.000 \mathrm{~g} / \mathrm{mol} ; \mathrm{M}_{\mathrm{w}} / \mathrm{M}_{\mathrm{n}}=2,5\right)$ e Teste $\left.6\left(\mathrm{M}_{\mathrm{w}}=282.000 \mathrm{~g} / \mathrm{mol} ; \mathrm{M}_{\mathrm{w}} / \mathrm{M}_{\mathrm{n}}=4,3\right)\right]$. A diminuição da massa molecular, do teste 3 para o teste 6 , pode estar relacionada ao aumento da temperatura do meio reacional, que acelera as reações de transferência e/ou terminação.

A análise do espectro obtido na região do infravermelho médio (Figura 1) permitiu observar as principais bandas de absorção de um polietileno (Teste 6) relativas às vibrações $v \mathrm{CH}_{2}, \delta \mathrm{CH}_{2}$ e rCH $\mathrm{C}_{2}$ à $2900,1470 \mathrm{e} 720 \mathrm{~cm}^{-1}$.

Os polietilenos obtidos são essencialmente lineares, como pode ser comprovado pelas suas temperaturas de fusão (Tabela 1) e pela fraca intensidade da banda de absorção, na região do infravermelho médio, a $1376 \mathrm{~cm}^{-1}$. Essa banda de absorção é característica das vibrações $\delta_{\mathrm{s}} \mathrm{CH}_{3}$ e traduz a presença de grupamentos metila na cadeia olefínica. Cabe destacar que esta banda pode se sobrepor a outras bandas devidas à absorção dos grupamentos $\mathrm{CH}_{2}$.

A Figura 2 mostra a ampliação da região entre $1400 \mathrm{e}$ $1330 \mathrm{~cm}^{-1}$ para os testes 3 e 6 . O espectro do teste 3 mostra uma banda mais intensa a $1376 \mathrm{~cm}^{-1}$, em relação ao teste 6 , coerente com o $\%$ de cristalinidade, respectivamente, de $61 \%$ e $73 \%$ (determinado por DSC).

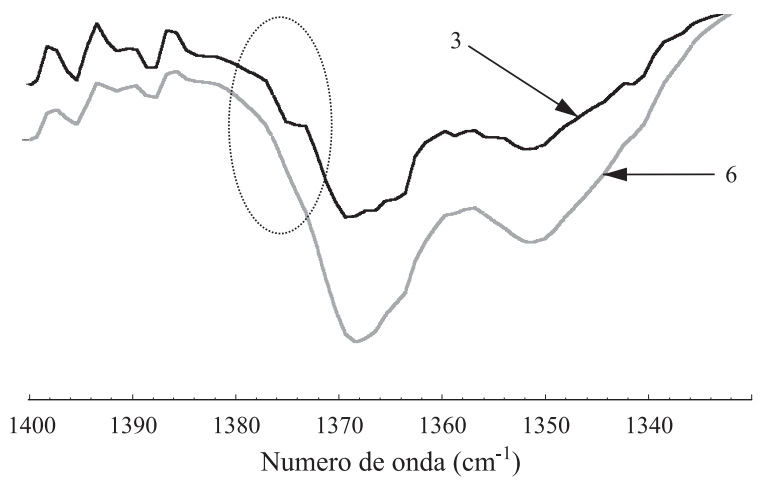

Figura 2. Espectro de absorção na região do infravermelho (1400 e 1330 $\mathrm{cm}^{-1}$ ) para os polietilenos obtidos nos testes catalíticos 3 e 6

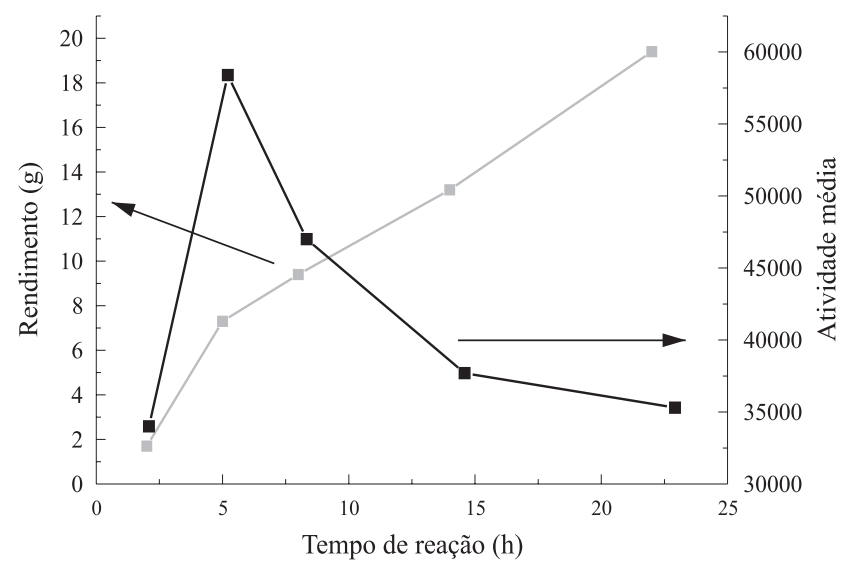

Figura 3. Variação dos rendimentos e das atividades médias em função do tempo (condições de polimerização: $100 \mathrm{~mL}$ tolueno, velocidade de agitação $=300 \mathrm{rpm}, 0,0125 \mathrm{mmol}$ de catalisador, pressão de etileno $=2 \mathrm{bar}, 20^{\circ} \mathrm{C}$, $\left.\mathrm{Al} / \mathrm{Cr}=20,20^{\circ} \mathrm{C}\right)$. Atividade média $=(\mathrm{g}$ polímero $/ \mathrm{h}$. bar.mol catalisador $)$.

O perfil cinético da reação de polimerização pode ser observado na Figura 3. A atividade passa por um máximo em torno de 5 horas de reação. Após esse tempo, o sistema começa a sofrer desativação, indicando uma instabilidade da espécie formada, após ativação com o cocatalisador.

\section{Copolimerização entre o Etileno e o Propileno e Caracterização dos Copolímeros Obtidos}

$\mathrm{O}$ sistema catalítico revelou-se inativo em relação à homopolimerização do propileno, porém foi possível obter copolímeros etileno-propileno. Um copolímero característico (teste 8) apresentou um ponto de fusão de $130{ }^{\circ} \mathrm{C}$ e um grau de cristalinidade de $45 \%$, coerentes com a existência de grupos metila (Me) provenientes da inserção do propileno na cadeia etilênica. A existência dessas metilas foi confirmada pelo espectro de $\mathrm{RMN}{ }^{13} \mathrm{C}$ (Me provenientes da inserção do propileno na cadeia etilênica marcadas com $*$ na Figura 4 ). As metilas terminais não aparecem muito intensas, devido provavelmente à alta massa molar dos polímeros.

No espectro de $\mathrm{RMN}{ }^{1} \mathrm{H}$ (Figura 5) observa-se, em torno de 1,20 ppm, o sinal correspondente aos grupamentos $\mathrm{C}-\mathrm{H}$ (metino) e a 0,94 ppm o sinal da metila $(d, M e)$, ambos característicos da inserção do propileno. A integração desses sinais,

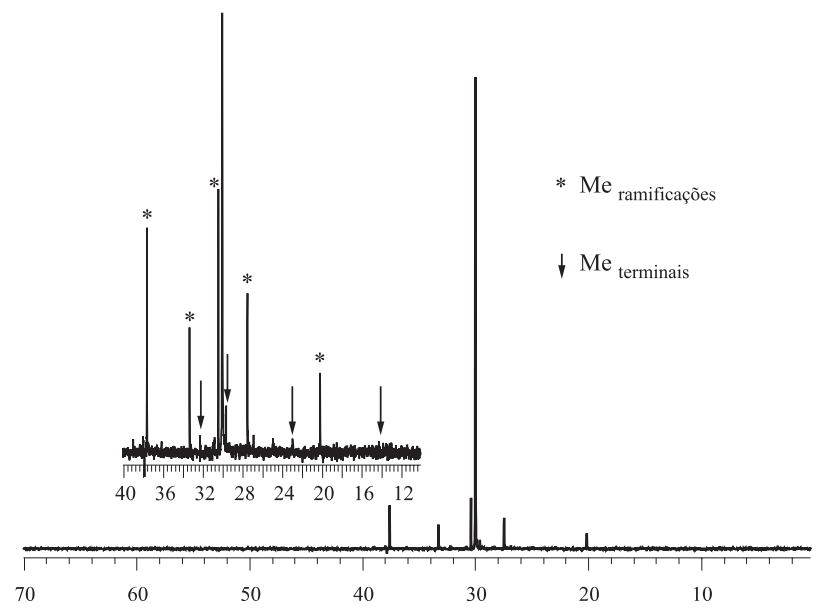

Figura 4. Espectro de $\mathrm{RMN}{ }^{13} \mathrm{C}$ do copolímero obtido no teste catalítico 8 


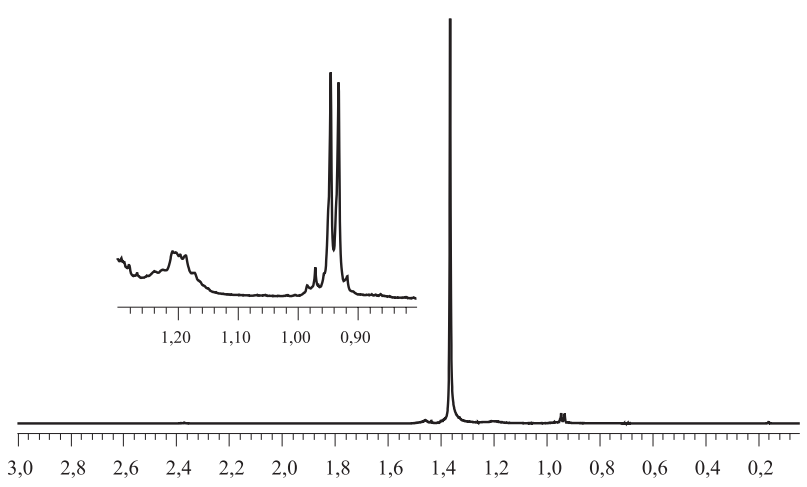

Figura 5. Espectro de $\mathrm{RMN}{ }^{1} \mathrm{H}$ do copolímero obtido no teste catalítico 8

em relação ao sinal principal da seqüência etilênica $(1,38$ ppm), revela a presença de $1 \mathrm{Me}$ para 17 grupos metileno, ou seja, uma taxa de inserção estimada em torno de $5 \%$ de propileno.

Cabe salientar que as extremidades insaturadas de cadeia, originadas de reações de terminação por eliminação $\beta$, não foram detectadas nos espectros obtidos por $\mathrm{RMN}^{13} \mathrm{C}$ e $\mathrm{RMN}{ }^{1} \mathrm{H}$ (entre 2,0 e 6,0 ppm).

\section{Conclusão}

A associação do ligante $t$-Bu- $\mathrm{N}_{4}$ ao $\mathrm{Cr}$ (III) levou à obtenção de um novo catalisador para polimerização de olefinas, cuja natureza não pode ser completamente elucidada. Os sistemas formados pela adição de cocatalisadores (MAO ou $\mathrm{Et}_{3} \mathrm{Al}_{2} \mathrm{Cl}_{3}$ ) são relativamente ativos e de vida longa, conduzindo à formação de polietilenos de massas molares elevadas. A homopolimerização do propileno não foi possível, porém foram obtidos copolímeros etileno-propileno. A atividade do sistema catalítico $t$ - $\mathrm{Bu}-\mathrm{N}_{4} \mathrm{CrCl}_{2}{ }^{+} \mathrm{Cl}^{-} / \mathrm{Et}_{3} \mathrm{Al}_{2} \mathrm{Cl}_{3}$ é mais alta que a do sistema $t-\mathrm{Bu}-\mathrm{N}_{4} \mathrm{CrCl}_{2}{ }^{+} \mathrm{Cl}^{-} / \mathrm{MAO}$.

\section{Agradecimentos}

Os autores agradecem a todos os colegas que contribuíram para a realização deste trabalho, ao CNRS pelo suporte financeiro (Programa Catalyse et catalyseurs pour l'industrie et l'environnement), à C.R.W. pelas análises SEC. S.P.M. agradece à CAPES pela bolsa de doutorado e a Politeno Ind. e Com. S/A.

\section{Referências Bibliográficas}

1. Theopold K.H.- Eur. J. Inorg. Chem., p. 15 (1998)

2. Thomas B.J., Theopold K.H.- J. Am. Chem. Soc., 110, p. 5902 (1988)

3. Coles M.P., Dalby C.I., Gibson V.C., Clegg W., Elsegood M.R.J.- J. Chem. Soc., Chem. Commun., , p.1709 (1995)

4. Gibson V.C., Maddox P.J., Newton C., Redshaw C., Solan G.A., White A.J.P., Williams D.J., J. Chem. Soc.- Chem. Commun., p.1651 (1998)

5. Kim W., Fevola M.J., Liable-Sands L.M., Rheingold A.L., Theopold K.H.- Organometallics, 17, p.4541 (1998)

6. Theopold K.H., Eur. J. Inorg. Chem., p.15 (1998)

7. Thomas B.J., Theopold K.H., J. Am. Chem. Soc., 110, p.5902 (1998)

8. Coles M.P., Dalby C.I., Gibson V.C., Clegg W., Elsegood M.R.J., J. Chem. Soc., Chem. Commun., p.1709 (1995)

9. Gibson V.C., Maddox P.J., Newton C., Redshaw C., Solan G.A., White A.J.P., Williams D.J., J. Chem. Soc., Chem. Commun., p.1651 (1998)

10. Kim W., Fevola M.J., Liable-Sands L.M., Rheingold A.L., Theopold K.H., Organometallics, 17, p.4541 (1998)

11. Meneghetti S.M.P. - "Polymérisation et Copolimérisation d'Olefines Catalysées par des Complexes de Métaux de Transition avc des Ligands Chélatants Polyazotés", Tese de Doutorado, Université Louis Pasteur, Strasbourg, França (2000)

12. Meneghetti S.M.P., Lutz P J, Fisher J., Kress J., Polyhedron, 20, p.2705 (2001)

13. Meneghetti S.M.P., Lutz P J, Kress J., Organometallics, 20, p.5050 (2001)

14. Perrin D.D., Armarengo W.L.F. Purification of Laboratory Chemicals, $3^{\text {rd }}$ Ed., Pergamon Press, New York, (1988)

15. Chen E.Y., Marks T.J., Chem. Rev., 100, p.1391 (2000)

Recebido: $10 / 10 / 02$

Aprovado: 17/02/03 